\title{
Probing the intermolecular interactions of PPARY-LBD with polyunsaturated fatty acids and their anti-inflammatory metabolites to infer most potential binding moieties
}

\author{
Shalini Muralikumar ${ }^{1 \dagger}$, Umashankar Vetrivel ${ }^{1 * \dagger}$, Angayarkanni Narayanasamy ${ }^{2}$ and Undurti N. Das $34^{3 *}$
}

\begin{abstract}
Background: PPARY is an isoform of peroxisome proliferator-activated receptor (PPAR) belonging to a super family of nuclear receptors. PPARy receptor is found to play a crucial role in the modulation of lipid and glucose homeostasis. Its commotion has been reported to play a significant role in a broad spectrum of diseases such as type 2 diabetes mellitus, inflammatory diseases, Alzheimer's disease, and in some cancers. Hence, PPARY is an important therapeutic target. Polyunsaturated fatty acids (PUFAs) and their metabolites (henceforth referred to as bioactive lipids) are known to function as agonists of PPARY. However, agonistic binding modes and affinity of these ligands to PPARY are yet to be deciphered.

Methods: In this study, we performed a comparative molecular docking, binding free energy calculation and molecular dynamics simulation to infer and rank bioactive lipids based on the binding affinities with the ligand binding domain (LBD) of PPARY.

Results: The results inferred affinity in the order of resolvin E1 > neuroprotectin D1 > hydroxy-linoleic acid > docosahexaenoic acid > lipoxin A4 > gamma-linolenic acid, arachidonic acid > alpha-linolenic acid > eicosapentaenoic acid > linoleic acid. Of all the bioactive lipids studied, resolvin E1, neuroprotectin D1 and hydroxy-linoleic acid showed significant affinity comparable to proven PPARy agonist namely, rosiglitazone, in terms of Glide XP docking score, H-bond formation with the key residues, binding free energy and stable complex formation with LBD favouring co-activator binding, as inferred through Molecular Dynamics trajectory analysis.
\end{abstract}

Conclusion: Hence, these three bioactive lipids (resolvin E1, neuroprotectin D1 and hydroxy-linoleic acid) may be favourably considered as ideal drug candidates in therapeutic modulation of clinical conditions such as type 2 DM, Alzheimer's disease and other instances where PPARY is a key player.

Keywords: PPARY, PUFA, Bioactive lipids, Docking, Helix12

\section{Background}

Peroxisome proliferator-activated receptor (PPAR) comprises of three isoforms, $\alpha, \beta$ and $\gamma$ belonging to a super family of nuclear receptors [1]. PPARs are ligand- activated transcription factors that regulate genes playing a

\footnotetext{
* Correspondence: vumashankar@gmail.com; Undurti@hotmail.com ${ }^{\dagger}$ Equal contributors

${ }^{1}$ Centre for Bioinformatics, Kamalnayan Bajaj Institute for Research in Vision and Ophthalmology, Vision Research Foundation, Sankara Nethralaya, Chennai 600 006, Tamil Nadu, India

${ }^{3}$ UND Life Sciences, 2020 S 360th St, \# K202, Federal Way, WA 98003, USA

Full list of author information is available at the end of the article
}

vital role over a broad spectrum of physiological and pathological conditions [2]. PPAR receptors are expressed by various tissues including muscles, hepatocytes, adipocytes and endothelial cells. Though the three isoforms of PPAR ( $\alpha, \beta$ and $\gamma$ ) share high level of sequence and structural similarity they are distinct in terms of expression and tissue distribution [3]. Molecular 3D structure of PPAR constitutes DNA binding domain at the $\mathrm{N}$-terminus and ligand binding domain (LBD) at the C-terminus. PPAR's interaction with its agonist leads to heterodimer formation with retinoid $\mathrm{X}$ 
receptor (RXR) [4]. PPAR-RXR heterodimer gets bounded at peroxisome proliferator response elements (PPREs) occupying the promoter region of target specific genes. Further, this process leads to recruitment of various transcriptional cofactors involved in the initiation of transcription process, thereby, triggering expression of several genes involved in diverse physiological and pathological processes [5-7]. Each PPAR subtype plays a unique physiological role in different tissues; however, all the three isoforms are well known to be involved in lipid and glucose homeostasis [8]. Of all the three isoforms, PPAR $\alpha$ and $\gamma$ are most extensively studied when compared to PPAR $\beta$.

PPAR $\alpha$ is predominantly expressed in tissues involved in metabolic activities of various tissues including muscles, heart, liver, intestine and brown adipose cells. PPAR $\alpha$ activation leads to a decrease in lipid levels. PPAR $\alpha$ receptor functions as a lipid sensor and helps in controlling energy combustion [9-12]. PPAR $\gamma$ is widely expressed in adipocytes, thereby, playing a crucial role in adipogenesis, lipid synthesis and in maintaining energy balance. PPAR $\gamma$ activation improves insulin sensitivity. In addition, PPAR $\gamma$ is expressed in spleen, large intestine, white and brown adipose tissues that may account for the involvement of these tissues in the pathobiology of type 2 Diabetes Mellitus and metabolic syndrome [10] $[13,14]$. PPAR $\beta$ is abundantly expressed in liver and abdominal adipose tissues by which it regulates blood cholesterol, glucose levels and influences fatty acid oxidation in cardiac and skeletal muscles $[15,16]$.

In the present study, we focussed on PPAR $\gamma$ receptor, as it plays a critical extensive role over broad spectrum of diseases such as type 2 diabetes mellitus, inflammatory diseases, Alzheimer's disease, and in some cancers [17-23]. PPAR $\gamma$ comprises a Y-shaped ligand binding domain (LBD), which is segmented into three arms, arm I, arm II and arm III. Arm I is extended towards helix12 (H12), known to be polar and widely conserved across the PPAR isoform [24-26]. It also harbors transcription activation function-2 (AF-2) at C-terminal region and is held in its active conformation by the hydrogen bonding network with Arm I favouring ligand binding [27, 28]. Arm II and Arm III are found to be less conserved compared to Arm I and are hydrophobic in nature [29]. It has been proposed that diverse ligands bind to PPAR $\gamma$ with different binding modes to LBD with most of the ligand binding scenarios displaying a hydrophilic interaction with Arm I region and hydrophobic interactions with either arm II or arm III regions [30].

It has been proposed that full agonists of PPARY reside over a large area of LBD of PPAR $\gamma$ with a U-shaped conformation and ideally comprising a polar head and a hydrophobic tail. The polar head of the full agonist forms a network of hydrogen bonds with the ARM-I residues (His449, His323, Ser289, Tyr327 and Tyr473) of PPARY side chains. The hydrogen bonds formed with these residues are responsible for the conformational change of $\mathrm{H} 12$ and activation of PPAR $\gamma$ activity [31]. In contrast, partial agonists activate PPAR $\gamma$ by an $\mathrm{H} 12$ independent mechanism, wherein, the key residues in LBD are completely different to that of the full agonists in that it leads to decrease in H12 stability, thereby affecting the coactivators binding, which, in turn, reduces the transcriptional activity of PPAR $\gamma[32,33]$. Most of the previous studies suggested that partial agonists form hydrogen bond with Ser342 of LBD [31, 33].

PPAR $\gamma$ agonists reduce lipid levels, enhance insulin sensitivity and thus, show anti-diabetic and antiinflammatory actions. PPAR $\gamma$, is well documented to be activated by a wide range of fatty acid molecules and their metabolites, of which PUFAs and its metabolites play a major role in exerting beneficial effects [34]. PUFAs are also reported to play significant roles in inflammatory and immune responses [35], lowering the levels of total cholesterol, Triglycerides and Low-density lipoproteins (LDL) [36, 37], in mediating apoptosis in colon cancer cells [38] and also shown to reduce the risk of early atrial fibrillation during post cardiac surgery [39]. Molecular modelling and structural bioinformatics studies serve as powerful and efficient tools for studying intermolecular interactions and dynamic behaviour of molecules in an in silico simulated conditions [40-42]. In the present study, we performed molecular docking of PPAR $\gamma$ against various PUFAs and its metabolites (henceforth called as bioactive lipids) [43], keeping cocrystal bound Rosiglitazone (BRL) as a reference structure (PDB ID: 4OF8). Further, the bound complexes were subjected to molecular dynamics simulation to compare the binding efficacies and to infer the agonistic binding modes of fatty acids, which are potential therapeutic molecules [44, 45], towards identification of the most potent and efficient bioactive lipid agonist targeting PPAR $\gamma$ (Fig. 1).

\section{Methods \\ Protein Preparation}

As a preliminary step, the crystal structure of PPAR $\gamma$ in complex with Rosiglitazone (PDB ID: 4O8F) at a resolution of $1.9 \AA$ was pre-processed using Protein preparation wizard of Schrödinger suite towards optimizing the stereochemistry by assigning proper bond order, removing steric clashes, adding hydrogen atoms, fixing the disulphide bonds, missing residues, atoms and loops. Further refinement was performed by adjusting the terminal chi rotation of Asparagine, Glutamine and Histidine residues. Optimal protonation states for Histidine residues were also assigned followed by the removal of unwanted hetero groups. Finally, energy minimization 


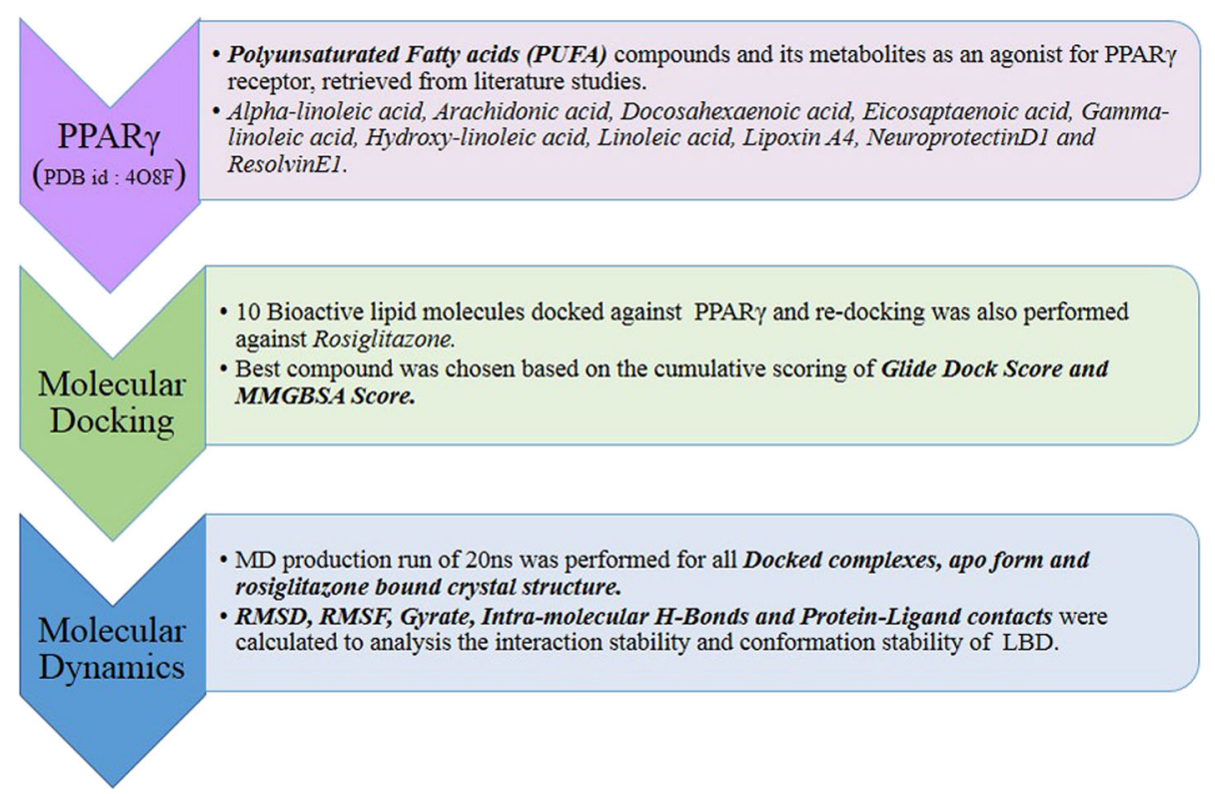

Fig. 1 Schematic representation of in silico study on PPARy agonists. (Note: PUFA molecule and its metabolites are collectively referred as bioactive lipids)

was performed with OPLS2005 to obtain the optimal geometry favourable for the commencement of plausible docking studies.

\section{Receptor grid generation}

The receptor grid was generated using the grid generation option of the Schrodinger suite across the PPARy LBD domain to perform a targeted docking study. The grid was fixed across residues that play a critical role at the three different arms of the LBD by setting the vanderwaals radii for the receptor with a scaling factor of $1.0 \AA$ and a partial cut off of $0.25 \AA$.

\section{Ligand Preparation}

To expedite the protein-ligand docking workflow, all the ligand molecules were optimized using Schrodinger Ligprep module by fixing the vanderwaals radii with the scaling factor of $0.80 \AA$ and a partial charge cut-off of $0.15 \AA$. Finally, the optimized compounds were energy minimized with OPLS 2005 as force field.

\section{Receptor- Ligand Docking}

Docking studies establish interactions between protein and ligand molecules, thereby, aids in identifying the most favourable binding pose forming a stable complex with significant binding affinity, as scored by the docking score [46]. In this study, protein-ligand docking was performed using Schrodinger Glide version [7.0] across the grid set over the LBD region. Here, a flexible docking was performed against 10 ligand molecules, in which the best binding pose among 100 generated poses for each molecule was determined based on the Glide XP docking score. A rigid Re-docking was also performed for the rosiglitazone (BRL) against its bound crystal structure by setting the grid across the LBD so as to infer the predictive efficacy.

\section{MMGBSA Scoring}

Molecular Mechanics Generalized Born Surface Area (MMGBSA) scoring was also performed for all the docked complexes to calculate the binding free energies by implementing the equation through Schrödinger Prime Module:

$$
\Delta \mathbf{G}_{\mathrm{GB}}=\Delta \mathbf{E}_{\mathrm{MM}}+\Delta \mathbf{G}_{\text {solv }}+\Delta \mathbf{G}_{\mathrm{SA}}
$$

Where electrostatic solvation energy $\left(\Delta \mathrm{G}_{\mathrm{GB}}\right)$ was calculated by using the GB Models, $\Delta \mathrm{E}_{\mathrm{MM}}$ is the difference between the minimized energies of ligand-protein complex and the total energies of protein and ligand in free form. $\Delta G_{\text {solv }}$ is the difference in the GBSA solvation energies of the ligand-receptor complex and the sum of the solvation energies of receptor and ligand in the unbound state. $\Delta \mathrm{G}_{\mathrm{SA}}$ is the difference in the surface area energies for the free receptor and the ligand. It is used to identify plausible binding conformations among the docked complexes in terms of binding free energy towards further stringent ranking of the complexes [47].

\section{Molecular Dynamics simulation}

Molecular dynamics simulation was performed for apo, co-crystal structure (PDB id: 4O8F) and for all the 
docked complexes using Desmond 3.6. The system was built using a cubical box solvated with Simple Point Charge (SPC) water model. Subsequently, the system was neutralized by adding $4 \mathrm{Na}^{+}$ions for apo and $5 \mathrm{Na}^{+}$ions for complexes at a concentration of $\sim 6.22 \mathrm{mM}$. Further, this system was energy minimized with OPLS2005 as force field [48]. SHAKE algorithm was applied to restrain the geometry of water molecules, bond lengths and angles of heavy atoms and to constrain covalent bonds during MD simulation [49]. Periodic Boundary Conditions (PBC) were applied to stimulate a continuous system [50] and Particle Mesh Ewald method (PME) was applied for long range electrostatics [51]. Further, the system was equilibrated with NPT ensemble by setting temperature and pressure parameter to $300 \mathrm{~K}$ and 1.0 Bar, respectively. Nose-Hoover chain and Martyna-Tobias-Klein was chosen as a coupling algorithm for temperature and pressure, respectively [52, 53]. Further, the equilibrated system with a total of 48,294 atoms was simulated for $20 \mathrm{~ns}$ (nanosecond) with a time step of $2 \mathrm{fs}$ (femtosecond) and trajectories were recorded after every 1.0 ps. The Root Mean Square Deviation (RMSD) was calculated for the backbone atoms and were graphically analysed at a time point scale [54, 55]. Similarly, root mean square fluctuation (RMSF) for each residue was also calculated to compare the major conformational changes in the residues between apo form and docked complex forms by keeping the rosiglitazone (BRL) bound crystal structure as a reference [56]. The radius of gyration was also calculated to infer the compactness of protein-ligand complexes for the comparison with apo form [57]. The 2D inter-molecular interaction plots depicting the complex stability during the MD run was also generated to infer the stability of all the complex structures.

\section{Results}

\section{Comparative Molecular Docking studies of PPARy with bioactive lipids}

Molecular Docking was performed for all the 10 bioactive lipid compounds against the PPAR $\gamma$-LBD. Glide XP dock score and MMGBSA binding free energy score were calculated for all receptor-ligand docked complexes, which revealed the scores to be in the range of -4.8 to $-9.9 \mathrm{kcal} / \mathrm{mol}$ and -71.147 to $-106.046 \mathrm{kcal} / \mathrm{mol}$ (Table 1), respectively. Further, 2D interaction maps with

Table 1 Receptor-Ligand docking results with Glide XP score, H-bonds and MMGBSA

\begin{tabular}{|c|c|c|c|c|}
\hline \multirow[t]{2}{*}{ Compounds } & \multirow{2}{*}{$\begin{array}{l}\text { XP } \\
\text { Glide } \\
\text { score } \\
\text { kcal/ } \\
\text { mol }\end{array}$} & \multicolumn{2}{|l|}{$\begin{array}{l}\text { XH-bond } \\
\text { Interactions }\end{array}$} & \multirow{2}{*}{$\begin{array}{l}\text { MMGBSA } \\
\text { score } \\
\mathrm{kcal} / \mathrm{mol}\end{array}$} \\
\hline & & Donor & Acceptor & \\
\hline \multirow[t]{4}{*}{ Rosiglitazone (BRL) } & \multirow[t]{4}{*}{-6.833} & Hie449:NH & BRL: O & \multirow[t]{4}{*}{-105.038} \\
\hline & & Tyr473:OH & BRL:HN & \\
\hline & & Ser289:OH & BRL: O & \\
\hline & & Hie323:NH & BRL: O & \\
\hline \multirow[t]{3}{*}{ Resolvin E1 (RsvE1) } & \multirow[t]{3}{*}{-9.900} & Hie449:NH & REV1: O & \multirow[t]{3}{*}{-106.046} \\
\hline & & Tyr327:HO & REV1: HO & \\
\hline & & Glu291: O & REV1: HO & \\
\hline \multirow[t]{2}{*}{ Neuroprotectin D1 (NPD1) } & \multirow[t]{2}{*}{-9.664} & Ser289:OH & NPD1: $\mathrm{O}^{-}$ & \multirow[t]{2}{*}{-102.57} \\
\hline & & Ser289:OH & NPD1: OH & \\
\hline \multirow[t]{2}{*}{ Hydroxy-linoleic acid (H-LA) } & \multirow[t]{2}{*}{-6.235} & Tyr473:OH & HLA:HO & \multirow[t]{2}{*}{-95.027} \\
\hline & & Ser289:OH & HLA: $\mathrm{O}^{-}$ & \\
\hline Docosahexaenoic acid (DHA) & -7.925 & Hie323:NH & AA: O & -89.785 \\
\hline \multirow[t]{2}{*}{ Lipoxin A4 (LXA4) } & \multirow[t]{2}{*}{-5.796} & Tyr327:OH & LXA4: HO & \multirow[t]{2}{*}{-87.547} \\
\hline & & Hie449:NH & LXA4: O & \\
\hline \multirow[t]{2}{*}{ Gamma-linoleic acid (GLA) } & \multirow[t]{2}{*}{-5.068} & Hie323:NH & GLA: $\mathrm{O}^{-}$ & \multirow[t]{2}{*}{-77.117} \\
\hline & & Ser289:OH & GLA: $O^{-}$ & \\
\hline Arachidonic acid (AA) & -7.040 & Hie323:NH & $A A: O$ & -76.586 \\
\hline Alpha-linoleic acid (AL) & -5.174 & Hie449:NH & $\mathrm{AL}: \mathrm{O}^{-}$ & -73.657 \\
\hline \multirow[t]{2}{*}{ Eicosapentaenoic acid (EPA) } & \multirow[t]{2}{*}{-7.126} & Hie323:NH & EPA: O & \multirow[t]{2}{*}{-71.147} \\
\hline & & Ser289:OH & EPA: O & \\
\hline Linoleic acid (LA) & -4.820 & Glu343:NH & $\left\llcorner A: O^{-}\right.$ & -78.026 \\
\hline
\end{tabular}

Compounds are ranked collectively based on XP glide score and MMGBSA score showing significance with reference ligand rosiglitazone (RsvE1 $>$ NPD1 $>$ HLA $>$ DHA $>$ LXA4 > GLA > AA > ALA > EPA > LA) 
a cut-off of $4 \AA$ for each docked complex was generated to visualize the intermolecular interactions. This inferred that all the compounds to be majorly stabilized by hydrogen bonds during complex formation (Fig. 2). The re-docking of Rosiglitazone to PPAR $\gamma$-LBD also showed an agreeable deviation of $0.4 \AA$, inferring the predictive accuracy. The 2D maps were further scrutinized for ligand contacts with the hotspot residues spanning Arm-I, Arm-II, Arm-III and AF-2 domain towards classifying potential full and partial agonists.

Molecular dynamics simulation of the docked complexes An unrestrained molecular dynamics simulation study of apo form and all docked complexes were performed to infer the backbone stability, residue fluctuations, structural compactness measured in terms of RMSD, RMSF, and $\mathrm{Rg}$, respectively. The RMSD trajectory revealed that all complexes to be stable during the entire production run with the system convergence at $\sim 15 \mathrm{~ns}$ (Fig. 4). The RMSF trajectory inferred maximum fluctuations at L1 (238-251) and L2 (260-276) regions across all the apo and docked complexes (Fig. 5a). Rg trajectory also revealed structural compactness in apo and protein-ligand complexes (deviation within 1 Å) (Fig. 5b). A trajectory of Intra-molecular hydrogen bond counts (mean average of $210 \mathrm{H}$-bonds) was plotted for the entire production run. As expected, there was a gain in h-bonds in docked complexes in comparison with holo forms (Fig. 5c). For

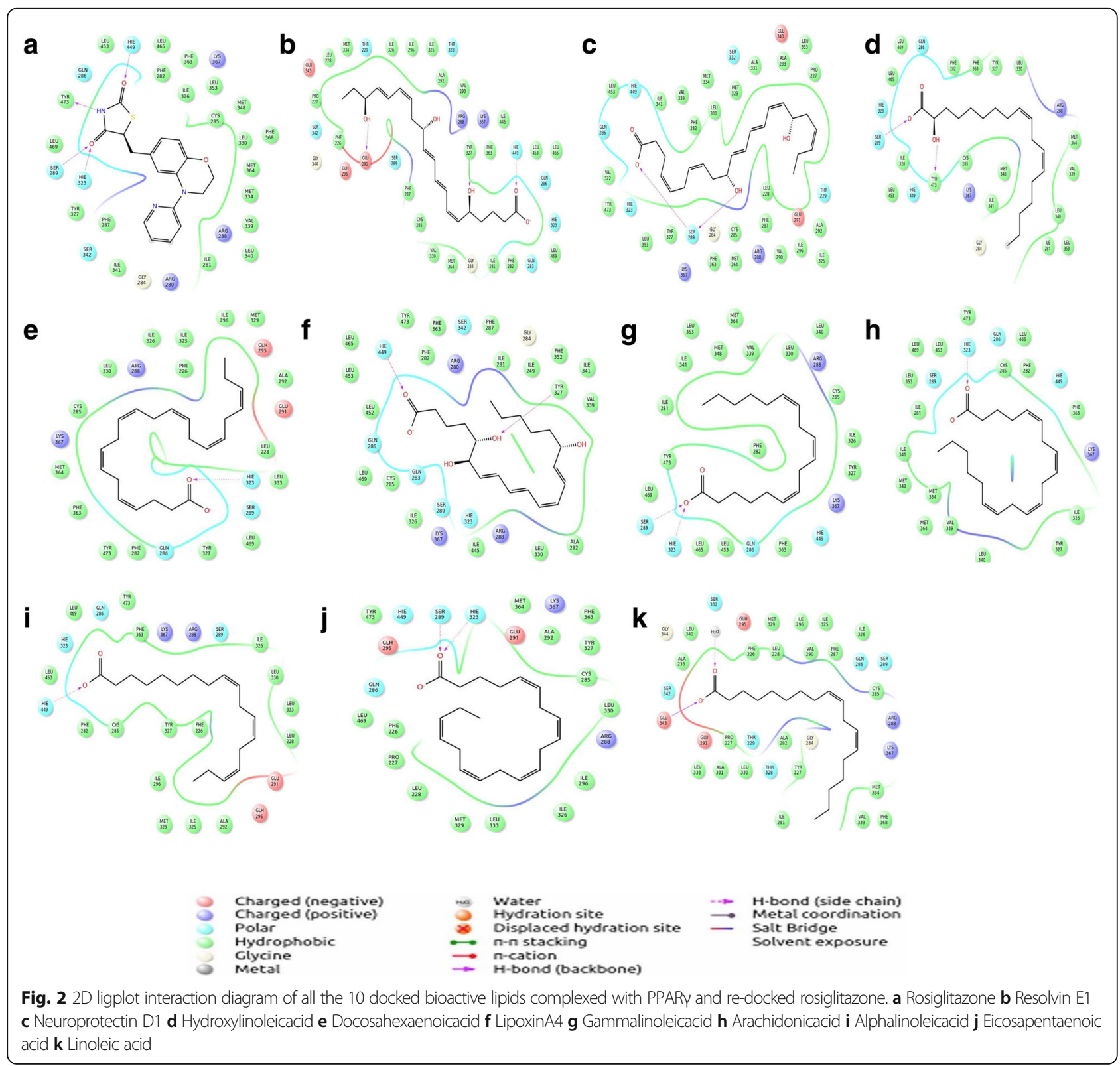




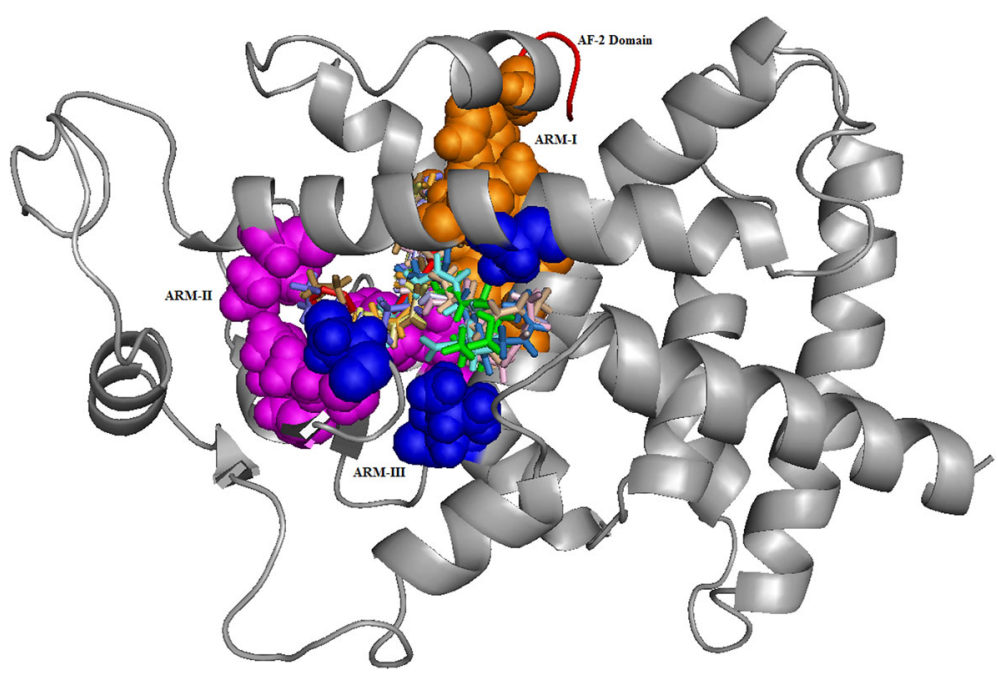

Fig. 3 All the 10 bioactive lipids were found to be docked within the three arms of the Ligand Binding Domain (LBD). All the ligands are represented as sticks with different colours (BRL: red, RsvE1: green, NPD1: sky blue, H-LA: pale green, DHA: light blue, LXA4: sand, GLA: orange, AA: pale yellow, AL: wheat, EPA: light pink, LA: aquamarine). ARM I (orange) involving four key residues (Tyr ${ }^{473}, \mathrm{His}^{323}, \mathrm{His}^{449}, \mathrm{Ser}^{289}$ and $\left.\mathrm{Tyr}^{327}\right)$ and $^{32}$ AF-2 at the C-terminal end of LBD critical for co-activator binding pocket. ARM II (magenta) constitutes six residues (Met ${ }^{364}, \|^{281}$, Met ${ }^{348},{ }^{2}{ }^{341}$ and Lys ${ }^{367}$ ) and ARM III (blue) with $\left(\right.$ Ala $^{292}$, Leu ${ }^{333} \&$ Ser $^{342}$ )

the top three ranking hits, Protein-ligands contact bar charts were plotted, which inferred the contribution of hotspot residues in establishment of intermolecular contacts, and was majorly found to be h-bond mediated (Fig. 6).

\section{Discussion}

\section{Receptor-Ligand Docking analysis}

Molecular Docking studies were subsequently performed for all the bioactive lipids against the assigned grid surface on the protein. The docked complexes were analysed for the Glide XP score, MMGBSA score and Hbond interactions, to collectively infer the ligand binding affinity [58, 59]. The Glide receptor-ligand docking results are tabulated in (Table 1). Among the docked complexes, Resolvin E1 (RsvE1), neuroprotectin D1 (NPD1) and hydroxy-linoleic acid (H-LA) showed significant affinity compared to other compounds in terms of XP dock score of $-9.900 \mathrm{kcal} / \mathrm{mol}, \quad-9.664 \mathrm{kcal} / \mathrm{mol}$ and $-6.235 \mathrm{kcal} / \mathrm{mol}$, along with a relative MMGBSA score of $-106.046 \mathrm{kcal} / \mathrm{mol},-102.57 \mathrm{kcal} / \mathrm{mol}$ and $-95.027 \mathrm{kcal} /$ mol, respectively. RsvE1 displayed strong intermolecular interactions, by forming three $\mathrm{H}$-bonds with the key side chain amino acids of LBD: amino group of Hie449 (H11), hydroxyl group of Tyr327 and Oxyl group of Glu291. NPD1 showed two H-bonds with the side chain of Ser289 and H-LA formed two $\mathrm{H}$-bond interactions with the $\mathrm{OH}$ group of side chain amino acids Tyr473 (H12) and Ser289 (H3), respectively. Moreover, RsvE1, NPD1 and H-LA also showed a significant scoring comparable to that of the reference ligand rosiglitazone (BRL), both in terms of docking score as well as MMGBSA score. Rosiglitazone on re-docking with the crystal structure yielded a XP dock score of $-6.833 \mathrm{kcal} / \mathrm{mol}$ and MMGBSA score of $-105.038 \mathrm{kcal} / \mathrm{mol}$. The glide re-docked complex of rosiglitazone (BRL) showed a RMSD of 0.446 Á on structural alignment with the native PPAR $\gamma$-Rosiglitazone cocrystallized structure, inferring the predictive accuracy of the method implemented (Table 1).

RsvE1, NPD1 and H-LA formed hydrogen bonds with the arm-I residues Hie449 (H11), Ser289(H3) and Tyr327, which are reported to be important for producing full activity of the compound by direct stabilization of $\mathrm{H} 12$ helix and are responsible for the transactivation activity of PPAR $\gamma[24,31,60,61]$. HLA also formed a hydrogen bond with Tyr473 (H12) located on the arm I that harbours transcription activation function-2(AF-2), which is obligatory for ligand binding and as well as in complimenting PPAR $\gamma$ function [27]. Tyr473 is also hypothesized to play a crucial role in AF-2 stabilization, as it occupies helix12 which closes the ligand binding site upon ligand binding. This activity of helix 12 favours in the reduction of conformational fluctuations that sets an optimal LBD structure for co-activator binding. It has also been reported that mutation at Tyr473 leading to the loss of agonistic activation by the ligands.

Alpha-linoleic acid (ALA) showed a Glide XP dock score of $-5.174 \mathrm{kcal} / \mathrm{mol}$ by forming a single $\mathrm{H}$-bond with the side chain of Hie449 (H11). Arachidonic acid (AA) and docosahexaenoic acid (DHA) formed a single $\mathrm{H}$-bond with the side chain of Hie323 with an XP dock score of $-7.040 \mathrm{kcal} / \mathrm{mol}$ and $-7.925 \mathrm{kcal} / \mathrm{mol}$, 


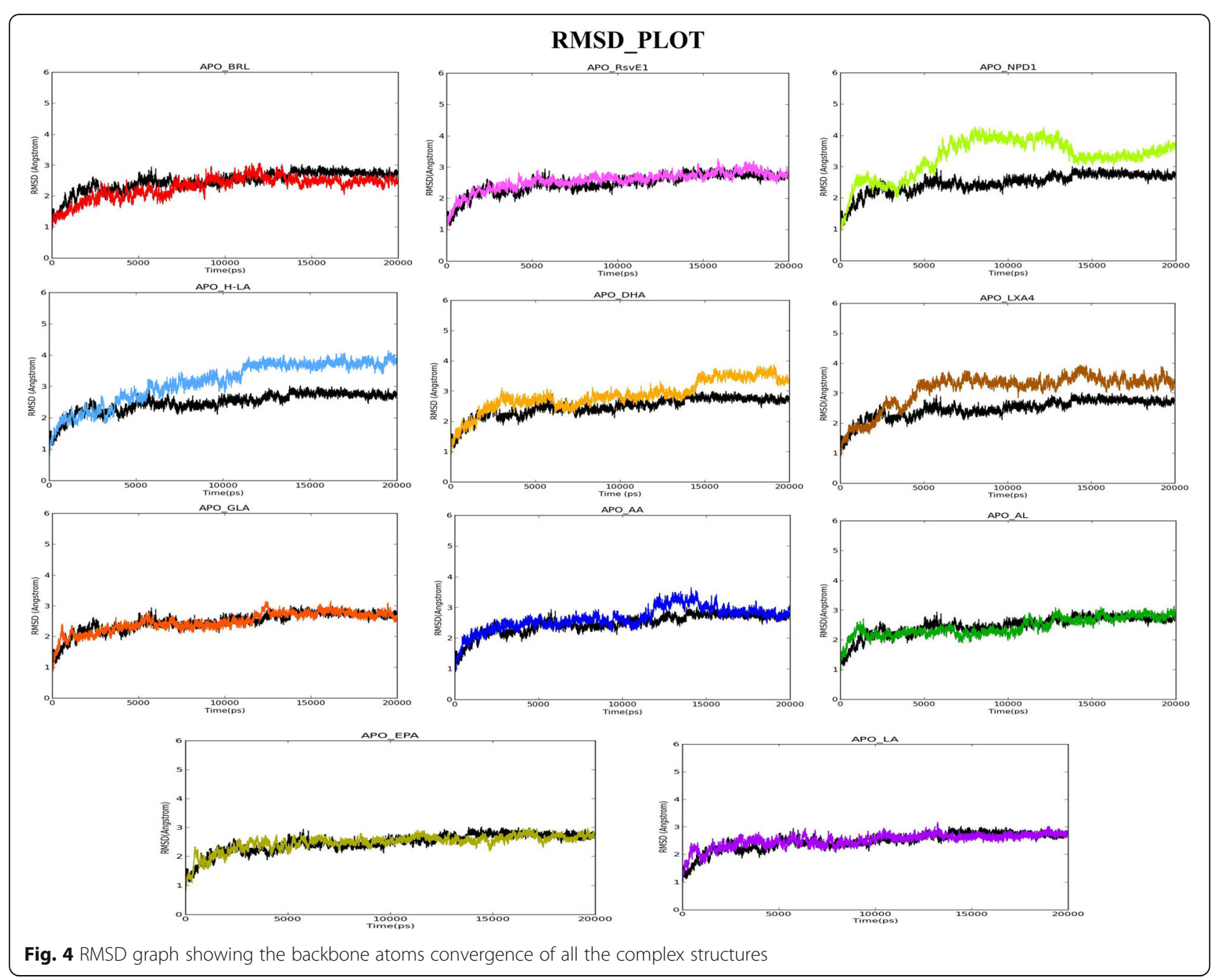

respectively. Eicosapentaenoic acid (EPA) and gammalinoleic acid (GLA) displayed a XP dock score of $-7.126 \mathrm{kcal} / \mathrm{mol}$ and $-5.068 \mathrm{kcal} / \mathrm{mol}$ by forming two hydrogen bonds with the side chain residues of Hie323 and Ser289, respectively. Lipoxin A4 (LXA4) with a docking XP score of $-5.796 \mathrm{kcal} / \mathrm{mol}$, displayed two hydrogen bond interactions with the active site residues Hie 449 and Tyr327. Linoleic acid (LA) showed the least XP dock score of $-4.820 \mathrm{kcal} / \mathrm{mol}$ among all the docked bioactive lipids compounds by forming an $\mathrm{H}$-bond with the back bone of Glu342 (Table 1).

The ligand interactions with ARM-I side chains of Hie323 (H3), Hie449 (H11), Ser289(H3) and Tyr327 of LBD is reported to play a significant role in enhancing agonistic activity as it is found to indirectly influence the H12 stabilization [24, 31, 60, 61]. Of the 10 docked complexes, 9 bioactive lipids are predicted to act as full agonists for PPAR $\gamma$ by forming an $\mathrm{H}$-bond interaction with the ARM-I hydrophilic cavity residues (Hie 323, Hie 449, Tyr473, Ser289 and Tyr327). Linoleicacid has been found to be a partial agonist for PPARY as it formed H-bond interactions with Glu343 of ARM-III [24]. The superimposition of all ten ligand molecules (bioactive lipids studied in the present study) along with the rosiglitazone as a reference ligand, inferred all the ligands to be well bound within the three arms of agonist binding domain by interacting with key residues (Figs. 2 and 3) [32, 62].

\section{Molecular dynamics studies of the protein-ligand complexes}

The RMSD trajectory of the backbone atoms of apo form and all the complex structures showed a convergence after a time frame ranging from $\sim 13,000 \mathrm{ps}$ to $\sim$ 15,000 ps with a maximum mean value of $\sim 3.5 \AA$ and S.D of $0.675 \AA$ (Fig. 4). The RMSF fluctuation has reached the maximum peak limit of 6-7⿵́ at the residues residing over the lengthy loop region (Loop1 with 14 residues and Loop2 with 17 residues) and remaining 


residues showed fluctuations within $1 \AA$. The H12 (Tyr473) residue showed a slightly higher fluctuation in the apo form compared to all the other protein-ligand complexes. This corroborates with the hypothesis that upon ligand binding to $\mathrm{H} 12$ hotspot to confer closure of LBD, thereby stabilizes the LBD conformation in the ligand bound state (Fig. 5a) [27]. The apo form and all the 10 (bioactive lipids) complexes were found to be compact; by displaying a radius of gyration less than $\sim 1.3$

(Fig. 5b). The intra-molecular H-bond graph also inferred no major loss in the protein secondary structure conformations, thereby, reinforcing that the proteinligand complex structures to be well stabilized (Fig. 5c). However, the protein complexed with the ligands Rsv E1, NPD1 and H-LA were found to establish strong protein-ligand contacts by constituting plausible intermolecular $\mathrm{H}$-bonds with the active site residues of the LBD domain throughout the period of simulation. RsvE1, NPD1 and H-LA displayed a consistent and plausible $\mathrm{H}$-bond interactions with the critical residues of ARM-1 (Hie323, Tyr327, Hie449, Lys367 and Tyr473) for $\sim 50 \%$ during the production run (Fig. 6a-c). The three bioactive lipids (RsvE1, NDP1 and H-LA) interacting with two conserved side chain amino acids Tyr327 and Lys367, are reported for favouring the formation of $\mathrm{H}$-bonds at the Keto-group of the ligands. These amino acid side chains are known to play a vital role in determining the specificity of ligands that can efficiently couple with the receptor [62] (Fig. 6a and b). It is noteworthy that these residues of arm-1 (His323, His449, Tyr473, Tyr327 and Lys367) are hypothesized for indirect and direct stabilisation of H12 (Tyr473), thereby favouring for co-activator binding and for contributing towards the transactivity of PPAR $\gamma$.

\section{Conclusion}

In the present study, we performed a molecular docking analysis with bioactive lipid compounds which are documented to be agonists of PPARY [30]. All the 10 docked complexes were found to be well bound within the three different arms of LDB. Thereby in-order to perform a further comparative study on the protein-ligand interaction stability and the conformational stability of the LBD, molecular dynamics studies were carried out for apo form, LBD co-crystallized with rosiglitazone and for all the docked complexes with bioactive lipids.

The cumulative analysis based on the Glide score, Prime-MMGBSA free binding energy score and MD trajectories analysis infer that RsvE1, NDP1 and H-LA to be the best docked compounds as these three showed relatively a significant score to that of the re-docking score of the reference ligand Rosiglitazone in terms of glide XP score, MMGBSA score and $\mathrm{H}$-bond forming pattern with the key residues (Table 1). The MD trajectory also reinforce that RsvE1, NDP1 and H-LA to be the best compounds based upon the RMSF graph (Fig. 5a), which clearly depicts that upon RsvE1, NDP1 and H-LA ligands binding to the LBD, it displays a much lesser fluctuation at $\mathrm{H} 12$ region in comparison to that of the reference ligand Rosiglitazone (BRL) and thereby maintaining a stable LBD structure for coactivator binding.

The protein-ligand contacts of the RsvE1, NDP1 and H-LA with PPARy throughout the production run of $20 \mathrm{~ns}$ also corroborates well by displaying a strong intermolecular interaction with the key active site residues of the LBD domain over $\sim 50 \%$ of the simulation time, which confirms the stability upon the ligand binding and its intactness with the receptor.

Moreover, RsvE1 is found to mimic the insulin sensitizing and anti-steatotic activities of omega-3-PUFAs, and also found to induce adiponectin expression similar to rosiglitazone [63]. NPD1 interacting with the PPARy is hypothesized to be play a significant role in suppressing neuroinflammation and thus, is considered to be of benefit in some neurodegenerative diseases [64]. Hydroxy-linoleic acid (H-LA) is reported to have anti-proliferative action on tumor cells [65]. In the present study, we have ranked all these PUFA compounds collectively based on the Glide docking score, $\mathrm{H}$-bonding formation with the key residues, Prime MMGBSA score and MD simulation trajectory analyses and observed that their relative PPAR $\gamma$ agonist activity to be as follows: RsvE1 $>$ NPD1 $>$ H-LA $>$ DHA $>$ LXA4 $>$ GLA $>$ AA $>$ ALA $>$ EPA $>$ LA. However, Further experimental studies need to be performed to verify the results obtained in the present bioinformatics analysis. Such studies could include the assessment of ability of these bioactive lipids in the pathobiology of obesity, insulin resistance, type $2 \mathrm{DM}$, metabolic syndromes and cancer.

\section{Abbreviations \\ AA: Arachidonic acid; AF-2: Transcription Activation Function-2; AL: Alpha- linoleic acid; BRL: Rosiglitazone; DHA: Docosahexaenoic acid; EPA: Eicosapentaenoic acid; GLA: Gamma-linoleic acid; H-LA: Hydroxy-linoleic acid; LA: Linoleic acid; LBD: Ligand Binding Domain; LDL: Low density lipoprotein; LXA4: Lipoxin A4; MD: Molecular dynamics; MMGBSA: Molecular Mechanics Generalized Born Surface Area; NPD1: Neuroprotectin D1; OPLS: Optimized Potentials for Liquid Simulations; PBC: Periodic boundary conditions; PME: Particle Mesh Ewald; PPARa: Peroxisome proliferator- activated receptor alpha; PPARB: Peroxisome proliferator-activated receptor beta; PPARy: Peroxisome proliferator-activated receptor gamma; PUFAs: Poly unsaturated fatty acids; Rg: Radius of gyration; RMSD: Root mean square deviation; RMSF: Root mean square fluctuation; RsvE1: Resolvin E1; SPC: Simple point charge; XP: Extra precision score}

\section{Acknowledgments}

The authors thank Indian Council of Medical Research, New Delhi for providing Bioinformatics infrastructure to conduct this study.

\section{Funding}

Indian Council of Medical Research, New Delhi -Project sanction order ICMR. No. (BIC/12(7)/2012)). The funding organisation had no role in study design, data collection and analysis, decision to publish, or preparation of the manuscript. 


\section{Availability of data and materials}

The dataset supporting the conclusions of this article is included within the article.

\section{Authors' contributions}

UV and SM planned and performed the structural bioinformatics studies and literature compilation. UND originated the idea and proposed the hypothesis of interactions between PUFAs and anti-inflammatory metabolites to PPARY, initiated the bioinformatics studies and supervised the study along UV. AN helped on enriching the biochemical literature and result interpretation in the study. All authors drafted the manuscript, read and approved the final version.

\section{Competing interests}

The authors declare that they have no competing interests.

\section{Consent for publication}

Not Applicable.

\section{Ethics approval and consent to participate}

Not Applicable.

\begin{abstract}
Author details
${ }^{1}$ Centre for Bioinformatics, Kamalnayan Bajaj Institute for Research in Vision and Ophthalmology, Vision Research Foundation, Sankara Nethralaya, Chennai 600 006, Tamil Nadu, India. ${ }^{2}$ Department of Biochemistry and Cell Biology, Kamalnayan Bajaj Institute for Research in Vision and Ophthalmology, Vision Research Foundation, Sankara Nethralaya, Chennai 600 006, Tamil Nadu, India. ${ }^{3}$ UND Life Sciences, 2020 S 360th St, \# K202, Federal Way, WA 98003, USA. ${ }^{4}$ BioScience Research Centre, GVP College of Engineering, Visakhapatnam 530048, India.
\end{abstract}

Received: 7 December 2016 Accepted: 28 December 2016 Published online: 21 January 2017

\section{References}

1. Guasch L, Sala E, Castell-Auvi A, Cedo L, Liedl KR, Wolber G, et al. Identification of PPARgamma partial agonists of natural origin (I): development of a virtual screening procedure and in vitro validation. PLoS One. 2012;7:e50816. doi:10. 1371/journal.pone.0050816.

2. Grygiel-Gorniak B. Peroxisome proliferator-activated receptors and their ligands: nutritional and clinical implications-a review. Nutr J. 2014;13:17. doi:10.1186/1475-2891-13-17.

3. Willson TM, Brown PJ, Sternbach DD, Henke BR. The PPARs: from orphan receptors to drug discovery t. J Med Chem. 2000;43:527-50. doi:10.1021/ jm990554g.

4. Berger J, Moller DE. The mechanisms of action of PPARs. Annu Rev Med 2002:53:409-35. doi:10.1146/annurev.med.53.082901.104018.

5. Yu S, Reddy JK. Transcription coactivators for peroxisome proliferatoractivated receptors. Biochim Biophys Acta. 1771;2007:936-51. doi:10.1016/j. bbalip.2007.01.008

6. Feige JN, Auwerx J. Transcriptional coregulators in the control of energy homeostasis. Trends Cell Biol. 2007;17:292-301. doi:10.1016/j.tcb.2007.04.001.

7. Wang L, Waltenberger B, Pferschy-Wenzig E, Blunder M, Liu X, Malainer C, et al. Natural product agonists of peroxisome proliferator-activated receptor gamma (PPARgamma): a review. Biochem Pharmacol. 2014;92:73-89. doi:10. 1016/j.bcp.2014.07.018.

8. Sertznig P, Seifert M, Tilgen W, Reichrath J. Present concepts and future outlook: function of peroxisome proliferator-activated receptors (PPARs) for pathogenesis, progression, and therapy of cancer. J Cell Physiol. 2007;212:1-12. doi:10.1002/jcp. 20998.

9. Neschen S, Morino K, Dong J, Wang-Fischer Y, Cline GW, Romanelli AJ, et al. $n-3$ Fatty acids preserve insulin sensitivity in vivo in a peroxisome proliferator-activated receptor-alpha-dependent manner. Diabetes. 2007;56: 1034-41. doi:10.2337/db06-1206.

10. Delerive P, Furman C, Teissier E, Fruchart J, Duriez P, Staels B. Oxidized phospholipids activate PPARalpha in a phospholipase A2-dependent manner. FEBS Lett. 2000;471:34-8.

11. Kliewer SA, Sundseth SS, Jones SA, Brown PJ, Wisely GB, Koble CS, et al. Fatty acids and eicosanoids regulate gene expression through direct interactions with peroxisome proliferator-activated receptors alpha and gamma. Proc Natl Acad Sci U S A. 1997;94:4318-23.
12. Lo Verme J, Fu J, Astarita G, La Rana G, Russo R, Calignano A, Piomelli D. The nuclear receptor peroxisome proliferator-activated receptor-alpha mediates the anti-inflammatory actions of palmitoylethanolamide. Mol Pharmacol. 2005;67:15-9. doi:10.1124/mol.104.006353.

13. Sheu S, Kaya T, Waxman DJ, Vajda S. Exploring the binding site structure of the PPAR gamma ligand-binding domain by computational solvent mapping. Biochemistry. 2005;44:1193-209. doi:10.1021/bi048032c.

14. Medina-Gomez G, Gray SL, Yetukuri L, Shimomura K, Virtue S, Campbell M, et al. PPAR gamma 2 prevents lipotoxicity by controlling adipose tissue expandability and peripheral lipid metabolism. PLoS Genet. 2007;3:e64. doi:10.1371/journal.pgen.0030064.

15. Wang Y, Lee C, Tiep S, Yu RT, Ham J, Kang H, Evans RM. Peroxisomeproliferator-activated receptor delta activates fat metabolism to prevent obesity. Cell. 2003;113:159-70.

16. Stephen RL, Gustafsson MCU, Jarvis M, Tatoud R, Marshall BR, Knight D, et al. Activation of peroxisome proliferator-activated receptor delta stimulates the proliferation of human breast and prostate cancer cell lines. Cancer Res. 2004; 64:3162-70.

17. Jialal I, Smith G. Managing the dyslipidemia of metabolic syndrome: beyond statin therapy. Metab Syndr Relat Disord. 2012;10:159-60. doi:10.1089/met.2012.1500.

18. Wang Y, Jacome-Sosa MM, Ruth MR, Lu Y, Shen J, Reaney MJ, et al. The intestinal bioavailability of vaccenic acid and activation of peroxisome proliferator-activated receptor-alpha and -gamma in a rodent model of dyslipidemia and the metabolic syndrome. Mol Nutr Food Res. 2012;56: 1234-46. doi:10.1002/mnfr.201100517.

19. Wilding JPH. PPAR agonists for the treatment of cardiovascular disease in patients with diabetes. Diabetes Obes Metab. 2012;14:973-82. doi:10.1111/j. 1463-1326.2012.01601.x

20. Wayman NS, Hattori Y, McDonald MC, Mota-Filipe H, Cuzzocrea S, Pisano B, et al. Ligands of the peroxisome proliferator-activated receptors (PPARgamma and PPAR-alpha) reduce myocardial infarct size. FASEB J. 2002;16: 1027-40. doi:10.1096/fj.01-0793com.

21. Bolden A, Bernard L, Jones D, Akinyeke T, Stewart LV. The PPAR gamma agonist troglitazone regulates Erk 1/2 phosphorylation via a PPARgammaIndependent, MEK-dependent pathway in human prostate cancer cells. PPAR Res. 2012;2012:929052. doi:10.1155/2012/929052.

22. Robbins GT, Nie D. PPAR gamma, bioactive lipids, and cancer progression. Front Biosci (Landmark Ed). 2012;17:1816-34.

23. Segawa Y, Yoshimura R, Hase T, Nakatani T, Wada S, Kawahito Y, et al. Expression of peroxisome proliferator-activated receptor (PPAR) in human prostate cancer. Prostate. 2002;51:108-16. doi:10.1002/pros.10058.

24. Guasch L, Sala E, Valls C, Blay M, Mulero M, Arola L, et al. Structural insights for the design of new PPARgamma partial agonists with high binding affinity and low transactivation activity. J Comput Aided Mol Des. 2011;25: 717-28. doi:10.1007/s10822-011-9446-9.

25. Fyffe SA, Alphey MS, Buetow L, Smith TK, Ferguson MAJ, Sorensen MD, et al. Recombinant human PPAR-beta/delta ligand-binding domain is locked in an activated conformation by endogenous fatty acids. J Mol Biol. 2006;356: 1005-13. doi:10.1016/j.jmb.2005.12.047.

26. Markt P, Schuster D, Kirchmair J, Laggner C, Langer T. Pharmacophore modeling and parallel screening for PPAR ligands. J Comput Aided Mol Des. 2007;21:575-90. doi:10.1007/s10822-007-9140-0.

27. Zoete V, Grosdidier A, Michielin O. Peroxisome proliferator-activated receptor structures: ligand specificity, molecular switch and interactions with regulators. Biochim Biophys Acta. 1771;2007:915-25. doi:10.1016/.j.bbalip.2007.01.007.

28. Gurula H, Loganathan T, Krishnamoorthy T, Vetrivel U, Samuel S. Virtual screening studies of seaweed metabolites for predicting PPARy agonists. Int J Pharm Pharmaceutical Sci. 2015;7:268-71.

29. Xu HE, Lambert MH, Montana VG, Parks DJ, Blanchard SG, Brown PJ, et al. Molecular recognition of fatty acids by peroxisome proliferator-activated receptors. Mol Cell. 1999;3:397-403.

30. Sundriyal S, Bharatam PV. Important pharmacophoric features of pan PPAR agonists: common chemical feature analysis and virtual screening. Eur J Med Chem. 2009;44:3488-95. doi:10.1016/j.ejmech.2009.01.024.

31. Farce A, Renault N, Chavatte P. Structural insight into PPARgamma ligands binding. Curr Med Chem. 2009;16:1768-89.

32. Bruning JB, Chalmers MJ, Prasad S, Busby SA, Kamenecka TM, He Y, et al. Partial agonists activate PPARgamma using a helix 12 independent mechanism. Structure. 2007;15:1258-71. doi:10.1016/.jstr.2007.07.014.

33. Lu I, Huang C, Peng Y, Lin Y, Hsieh H, Chen C, et al. Structure-based drug design of a novel family of PPARgamma partial agonists: virtual screening, 
X-ray crystallography, and in vitro/in vivo biological activities. J Med Chem. 2006;49:2703-12. doi:10.1021/jm051129s.

34. El Akoum S. PPAR Gamma at the Crossroads of Health and Disease: A Masterchef in Metabolic Homeostasis. Endocrinol Metab Synd. 2014. doi:10. 4172/2161-1017.1000126.

35. Bhagat U, Das UN. Potential role of dietary lipids in the prophylaxis of some clinical conditions. Arch Med Sci. 2015;11:807-18. doi:10.5114/aoms.2015. 53302.

36. Sahebkar A, Serban M, Gluba-Brzozka A, Mikhailidis DP, Cicero AF, Rysz J, Banach M. Lipid-modifying effects of nutraceuticals: An evidence-based approach. Nutrition. 2016;32:1179-92. doi:10.1016/j.nut.2016.04.007.

37. Banach M, Aronow WS, Serban M, Rysz J, Voroneanu L, Covic A. Lipids, blood pressure and kidney update 2015. Lipids Health Dis. 2015;14:167. doi: 10.1186/s12944-015-0169-0.

38. Zhang C, Yu H, Shen Y, Ni X, Shen S, Das UN. Polyunsaturated fatty acids trigger apoptosis of colon cancer cells through a mitochondrial pathway. Arch Med Sci. 2015;11:1081-94. doi:10.5114/aoms.2015.54865.

39. Mariscalco G, Sarzi Braga S, Banach M, Borsani P, Bruno VD, Napoleone M, et al. Preoperative $n-3$ polyunsatured fatty acids are associated with a decrease in the incidence of early atrial fibrillation following cardiac surgery. Angiology. 2010;61:643-50. doi:10.1177/0003319710370962.

40. Umashankar V, Gurunathan S. In Silico Tools for Molecular Modeling. In: Ballantyne B, Marrs TC, Syversen T, Casciano DA, Sahu SC, editors. General, Applied and Systems Toxicology. Chichester: Wiley; 2009. doi:10.1002/ 9780470744307.gat234.

41. Umashankar V, Gurunathan S. Chemoinformatics and its Applications. In: Ballantyne B, Marrs TC, Syversen T, Casciano DA, Sahu SC, editors. General, Applied and Systems Toxicology. Chichester: Wiley; 2009. doi:10.1002/ 9780470744307.gat222.

42. Umashankar VGS. DRUG DISCOVERY: AN APPRAISAL. Int J Pharm Pharmaceutical Sci. 2015;7:59-66

43. Vetrivel U, Ravichandran SB, Kuppan K, Mohanlal J, Das UN, Narayanasamy A. Agonistic effect of polyunsaturated fatty acids (PUFAs) and its metabolites on brain-derived neurotrophic factor (BDNF) through molecular docking simulation. Lipids Health Dis. 2012;11:109. doi:10.1186/1476-511X-11-109.

44. Ortuno Sahagun D, Marquez-Aguirre AL, Quintero-Fabian S, Lopez-Roa RI, Rojas-Mayorquin AE. Modulation of PPAR-gamma by Nutraceutics as Complementary Treatment for Obesity-Related Disorders and Inflammatory Diseases. PPAR Res. 2012;2012:318613. doi:10.1155/2012/318613.

45. Serhan CN, Yacoubian S, Yang R. Anti-inflammatory and proresolving lipid mediators. Annu Rev Pathol. 2008;3:279-312. doi:10.1146/annurev. pathmechdis.3.121806.151409.

46. Sandeep S, Priyadarshini V, Pradhan D, Munikumar M, Umamaheswari A. Docking and molecular dynamics simulations studies of human protein kinase catalytic subunit alpha with antagonist. J Clin Sci Res. 2012:15-23 doi: 10.15380/2277-5706.JCSR.12.005

47. Genheden S, Ryde U. The MM/PBSA and MM/GBSA methods to estimate ligand-binding affinities. Expert Opin Drug Discov. 2015;10:449-61. doi:10. 1517/17460441.2015.1032936

48. DuBay KH, Hall ML, Hughes TF, Wu C, Reichman DR, Friesner RA. Accurate force field development for modeling conjugated polymers. J Chem Theory Comput. 2012;8:4556-69. doi:10.1021/ct300175w.

49. Barth E, Kuczera K, Leimkuhler B, Skeel RD. Algorithms for constrained molecular dynamics. J Comput Chem. 1995;16:1192-209. doi:10.1002/jcc. 540161003.

50. Bulatov W, Rhee M, Cai W. Periodic boundary conditions for dislocation dynamics simulations in three dimensions. Mater Res Soc Proc. 2000;653:21-3.

51. Harvey MJ, de Fabritiis G. An implementation of the smooth particle mesh ewald method on GPU hardware. J Chem Theory Comput. 2009:5:2371-7. doi:10.1021/ct900275y.

52. Kleinerman DS, Czaplewski C, Liwo A, Scheraga HA. Implementations of Nose-Hoover and Nose-Poincare thermostats in mesoscopic dynamic simulations with the united-residue model of a polypeptide chain. J Chem Phys. 2008;128:245103. doi:10.1063/1.2943146.

53. Martyna GJ, Tobias DJ, Klein ML. Constant pressure molecular dynamics algorithms. J Chem Phys. 1994;101:4177. doi:10.1063/1.467468.

54. Damm KL, Carlson HA. Gaussian-weighted RMSD superposition of proteins: a structural comparison for flexible proteins and predicted protein structures. Biophys J. 2006;90:4558-73. doi:10.1529/biophysj.105.066654.
55. Maiorov VN, Crippen GM. Significance of root-mean-square deviation in comparing three-dimensional structures of globular proteins. J Mol Biol. 1994;235:625-34. doi:10.1006/jmbi.1994.1017.

56. Fuglebakk E, Echave J, Reuter N. Measuring and comparing structural fluctuation patterns in large protein datasets. Bioinformatics. 2012;28:243140. doi:10.1093/bioinformatics/bts445.

57. Lobanov MI, Bogatyreva NS, Galzitskaia OV. Radius of gyration is indicator of compactness of protein structure. Mol Biol (Mosk). 2008;42:701-6.

58. Vetrivel U, Muralikumar S, Mahalakshmi B, Lily Therese K, Madhavan HN, Alameen $\mathrm{M}$, Thirumudi I. Multilevel precision-based rational design of chemical inhibitors targeting the hydrophobic cleft of toxoplasma gondii Apical Membrane Antigen 1 (AMA1). Genomics Inform. 2016;14:53-61. doi:10.5808/GI.2016.14.2.53

59. John A, Sivashanmugam M, Umashankar V, Natarajan SK. Virtual screening, molecular dynamics, and binding free energy calculations on human carbonic anhydrase IX catalytic domain for deciphering potential leads. J Biomol Struct Dyn. 2016:1-14. doi:10.1080/07391102.2016.1207565.

60. Shashni B, Sharma K, Singh R, Sakharkar KR, Dhillon SK, Nagasaki Y, Sakharkar MK. Coffee component hydroxyl hydroquinone (HHQ) as a putative ligand for PPAR gamma and implications in breast cancer. BMC Genomics. 2013;14 Suppl 5:S6. doi:10.1186/1471-2164-14-S5-S6.

61. Itoh T, Fairall L, Amin K, Inaba Y, Szanto A, Balint BL, et al. Structural basis for the activation of PPARgamma by oxidized fatty acids. Nat Struct Mol Biol. 2008:15:924-31.

62. Tsukahara T, Tsukahara R, Yasuda S, Makarova N, Valentine WJ, Allison P, et al. Different residues mediate recognition of 1-O-oleyllysophosphatidic acid and rosiglitazone in the ligand binding domain of peroxisome proliferator-activated receptor gamma. J Biol Chem. 2006;281:3398-407. doi:10.1074/jbc.M510843200

63. Gonzalez-Periz A, Horrillo R, Ferre N, Gronert K, Dong B, Moran-Salvador E, et al. Obesity-induced insulin resistance and hepatic steatosis are alleviated by omega-3 fatty acids: a role for resolvins and protectins. FASEB J. 2009;23: 1946-57. doi:10.1096/fj.08-125674.

64. Palacios-Pelaez R, Lukiw WJ, Bazan NG. Omega-3 essential fatty acids modulate initiation and progression of neurodegenerative disease. Mol Neurobiol. 2010;41:367-74. doi:10.1007/s12035-010-8139-z.

65. Erazo T, Lorente M, Lopez-Plana A, Munoz-Guardiola P, Fernandez-Nogueira P, Garcia-Martinez JA, et al. The new antitumor drug ABTL0812 inhibits the Akt/mTORC1 axis by upregulating tribbles-3 pseudokinase. Clin Cancer Res. 2016;22:2508-19. doi:10.1158/1078-0432.CCR-15-1808.

\section{Submit your next manuscript to BioMed Central and we will help you at every step:}

- We accept pre-submission inquiries

- Our selector tool helps you to find the most relevant journal

- We provide round the clock customer support

- Convenient online submission

- Thorough peer review

- Inclusion in PubMed and all major indexing services

- Maximum visibility for your research

Submit your manuscript at www.biomedcentral.com/submit 